ÉGYPTE monde arabe

\section{Égypte/Monde arabe}

$12 \mid 2015$

Evolution des systèmes médiatiques après les révoltes arabes

\title{
Introduction : Le système hybride égyptien et le « chaos culturel»
}

\section{Enrico De Angelis}

\section{(2) OpenEdition}

\section{Journals}

Édition électronique

URL : https://journals.openedition.org/ema/3379

DOI : $10.4000 /$ ema.3379

ISSN : 2090-7273

Traduction(s) :

Introduction: The hybrid system of Egypt and "cultural chaos" - URL : https://journals.openedition.org/ ema/3398 [en]

Éditeur

CEDEJ - Centre d'études et de documentation économiques juridiques et sociales

Édition imprimée

Date de publication : 25 mars 2015

Pagination : $7-20$

ISBN : 9782905838858

ISSN : 1110-5097

Référence électronique

Enrico De Angelis, «Introduction : Le système hybride égyptien et le « chaos culturel » », Égypte/Monde arabe [En ligne], 12 | 2015, mis en ligne le 25 mars 2015, consulté le 07 juillet 2022. URL : http:// journals.openedition.org/ema/3379; DOI : https://doi.org/10.4000/ema.3379 


\section{Enrico De Angelis}

\section{INTRODUCTION}

\section{LE SYSTÈME HYBRIDE ÉGYPTIEN ET LE «CHAOS CULTUREL »}



e paysage médiatique arabe a subi ces dernières années de profondes transformations. Les révoltes de 2011 ont parfois contribué à accélérer ou à modifier ce processus.

Certaines de ces transformations ont lieu à l'échelle mondiale, telles que la diffusion accrue des technologies digitales et des social network, l'intégration croissante entre les anciens et les nouveaux médias, I'affirmation d'une sphère sociale et politique basée sur une connectivité plus importante, et enfin, la synergie entre internet et les mouvements de rue. D'autres phénomènes, en revanche, sont propres au monde arabe: le déclin des télévisions transnationales, du moins en termes de public, la tendance vers une communication centrée sur la politique interne plus que sur les questions internationales, et enfin le retour massif des mesures répressives et de la censure.

Plusieurs années seront nécessaires pour pouvoir évaluer de façon précise ces transformations et leur impact sur le futur de la région. II s'agit là en effet de processus complexes en évolution constante, tant sur le plan politique que médiatique: il suffit de se rappeler l'enthousiasme qu'avaient suscité les nouvelles technologies à l'époque des révoltes en 2011, enthousiasme auquel avaient rapidement succédé une indifférence excessive, voire la méfiance.

Du reste, le monde arabe n'est pas la seule région du monde où il soit si difficile d'interpréter les transformations à l'oeuvre dans le champ médiatique.

L'arrivée d'internet, la mondialisation de l'information et la convergence médiatique sont des phénomènes globaux, et le défi qu'ils 
représentent aujourd'hui nous contraint de repenser les approches théorico-méthodologiques à l'oeuvre dans le domaine des médias.

L'apparition du web 2.0, précédée de peu par celle des chaînes satellitaires, conduit à l'émergence d'un système de communication globale de plus en plus interconnecté, caractérisé par une surabondance de contenus et une diversité accrue des sources d'information. La digitalisation conduit à la production de nouvelles formes d'intersections entre différents médias et rend plus floue la distinction entre producteurs et consommateurs de l'information. Internet brouille les pistes entre sphère publique et privée, amenant ainsi à de profondes transformations dans le rapport entre politique et représentation, tout en rendant quasi obsolète la conception des médias comme " quatrième pouvoir ».

Il est extrêmement difficile d'identifier les tendances d'un environnement à ce point interconnecté et en continuelle évolution. Ce n'est pas un hasard si le sociologue Brian McNair parle de " chaos culturel » à propos du paysage médiatique contemporain, évoquant les difficultés croissantes des autorités politiques à imposer un contrôle hégémonique sur des agendas médiatiques de plus en plus imprévisibles et volatiles. ${ }^{1}$

Malgré l'attention qu'attache McNair à la perte de contrôle sur l'information de la part des gouvernements, l'actualité récente dans le monde arabe nous empêche de voir là un facteur jouant systématiquement en faveur des processus de démocratisation. Sami Ben Gharbeya, I'un des fondateurs du blog tunisien Nawaat, qualifie de "cascade d'information » la manière dont les activistes tunisiens sont parvenus à diffuser les récits révolutionnaires au niveau national et international, en s'appuyant sur des typologies de médias différentes. ${ }^{2}$ Toutefois, d'autres cas, comme celui de la Syrie, montrent que ce processus peut parfois se révéler bien plus opaque et moins efficace. De même, la pluralisation croissante de la circulation des contenus ne semble pas avoir favorisé la transition politique dans des pays tels que l'Egypte. Qu'ils soient plus ou moins démocratiques, les gouvernements semblent s'adapter de mieux en mieux aux logiques du « chaos culturel », du moins à l'heure actuelle.

Par ailleurs, il est encore bientôt pour évaluer les conséquences de ces transformations sur le long terme.

1. Brian McNair, 2006, Cultural Chaos. Journalism, News and Power in a Globalized World, Routledge, Londres.

2. Voir à ce propos, Ethan Zuckerman, "Civic Disobedience and the Arab Spring", My Heart is in Accra, 6 mai 2011 : http://www.ethanzuckerman. com/blog/2011/05/06/civic-disobedience-and-the-arab-spring/ 
C'est dans ce cadre qu' Egypte/Monde arabe entend proposer une série d'analyses portant sur les dynamiques des médias dans la région, se focalisant en particulier, mais pas seulement, sur le cas égyptien. On visera ici à apporter des clarifications sur certaines tendances ayant émergé ces dernières années, tout en restant conscient que le champ sur lequel porte la recherche continue d'être en évolution rapide et constante. En ce sens, les analyses présentées souhaitent éviter l'écueil de la simplification qui a caractérisé certaines interprétations de ces phénomènes, aussi bien dans le secteur journalistique que dans celui de la recherche. L'objectif sera donc ici de proposer des approches théoricométhodologiques inter-disciplinaires, permettant une compréhension plus profonde des changements à l'oeuvre actuellement, tout en offrant des contributions basées sur des recherches empiriques solides et de longues périodes d'observation.

L'importance accordée à ces analyses et le choix de consacrer un numéro de Egypte/Monde Arabe à ce sujet découlent de la prise de conscience que les médias doivent être considérés comme de véritables acteurs politiques, et non pas seulement comme un lieu de débat et de production de l'information.

La possibilité d'une transition vers des systèmes plus participatifs, plus ouverts et plus égalitaires continue de dépendre en grande partie des changements qui touchent le monde des médias dans la région. Par ailleurs, dans le cadre d'une médiatisation croissante de la politique, et de l'interpénétration progressive mais apparemment inévitable entre sphère publique et privée, et entre sphère sociale et politique, I'analyse des processus de communication devient cruciale pour les chercheurs dans d'autres disciplines. En ce sens, ce numéro entend également rapprocher des domaines académiques qui, tels que les sciences politiques et les médias, tendent souvent à s'ignorer réciproquement, faisant ainsi obstacle à une meilleure compréhension des développements politiques et sociaux dans la région.

\section{LE CAS ÉGYPTIEN: TENDANCES ET CONTRADICTIONS}

L'Egypte fournit un cas d'analyse des transformations récentes des médias particulièrement intéressant. D'une part, le système médiatique égyptien est généralement considéré comme l'un des systèmes les plus avancés dans la région. Le secteur privé compte plusieurs journaux et chaînes de télévisions relativement importants, tant en termes de budget que de public. La blogosphère égyptienne est la première à avoir attiré l'attention sur l'importance d'internet comme outil de dissension, et les pratiques qui la caractérisent ont servi d'exemple pour d'autres pays de la région. 
Et pourtant, ce système médiatique a montré ses limites ces dernières années, révélant l'ampleur des contradictions propres à ce système : au point que les médias se sont trouvés accusés d'être en partie responsables de l'échec, même temporaire, de la révolution égyptienne.

Le paysage médiatique égyptien fait donc office de laboratoire pour les nouvelles tendances, qui y émergent plus rapidement et de façon plus évidente que dans d'autres contextes régionaux.

Si certaines de ces tendances ont besoin de plus de temps pour pouvoir être soumises à l'analyse, d'autres sont déjà assez clairement identifiées pour pouvoir servir de point de référence dans les articles présentés dans ce numéro.

\section{Vers une communication politique plus locale et une décentralisation de l'information}

Dans la période immédiatement postérieure aux révoltes de 2011, une nouvelle sphère publique transnationale semble émerger dans le monde arabe. Grâce aux social network, les jeunes révolutionnaires de différents pays peuvent entrer en contact, échanger des informations, rejoindre des campagnes et comparer leurs expériences respectives. Cet espace public ne s'appuie plus sur les médias panarabes financés par les capitaux du Golfe, tout comme il se distingue également du panarabisme imposé dans les années 1960. Les "arabités numériques", comme les appelle Gonzalez-Quijano, sont ainsi l'expression d'un sentiment commun, partagé surtout au sein des jeunes générations, qui dépasse les frontières des Etats et est rarement représenté par les médias traditionnels. ${ }^{3}$

Cependant, le vent tourne pendant les années suivantes. Le public semble concentrer à nouveau son intérêt sur l'actualité nationale, se détournant des sujets qui occupaient traditionnellement I'agenda médiatique arabe telles que la question palestinienne et autres événements internationaux, y compris les "révolutions » voisines. La tendance semble transversale, étant commune à la fois aux médias traditionnels et aux nouveaux médias ainsi qu'à toutes les générations. Les sphères virtuelles, comme il est facile de l'observer en se promenant sur les social network, semblent se provincialiser de plus en plus. Même les nouveaux projets de journalisme digital apparus ces dernières années choisissent de se concentrer sur la dimension locale, parfois hyper-locale, plutôt que sur l'international. Bien qu'on parle déjà depuis longtemps de créer des synergies internationales entre diverses initiatives de journalisme digital " par le bas », rien de concret

3. Yves Gonzalez-Quijano, 2012, Arabités numériques. Le printemps du Web arabe, Sindbad, Paris. 
n'a encore été réalisé à ce jour qui aille dans cette direction. Quand aux médias nationaux traditionnels, privés ou non, leur couverture des événements internationaux n'est souvent qu'un outil au service d'un agenda politique interne bien précis. Les récits journalistiques diffusés à l'heure actuelle en Egypte sur la Syrie, la Libye ou encore l'Irak servent surtout à rappeler qu'une révolution peut coûter cher.

Le déclin des télévisions transnationales du Golfe est un phénomène plus significatif encore. Al-Jazeera surtout semble avoir perdu une partie importante de son public et avoir été reléguée au rang de "second choix», en tous cas lorsqu'il s'agit de suivre l'actualité locale. Le soutien apporté aux Frères Musulmans après 2011 ainsi que les erreurs de la chaîne dans la couverture médiatique d'autres événements, à commencer par la Syrie, ont contribué à éroder sa crédibilité. La perte de prestige de Al-Jazeera et de ses consoeurs, jointe à une demande croissante de nouvelles locales que les chaînes transnationales ont peine à fournir, ont poussé le public égyptien vers les télévisions nationales: OnTv, Dream Tv, al-Tahrir, al-Qahira wa al-Nas et CBC.

Cette dé-régionalisation des communications a inévitablement pour effet de faire évoluer l'impact des médias sur les processus sociopolitiques. Les télévisions telles que Al-Jazeera avaient en effet joué en 2011 un rôle fondamental dans la diffusion d'information sur les mobilisations, en particulier parmi les tranches de la société moins connectées aux nouvelles technologies. Toutefois, I'apparition de dizaines de plateformes de journalisme digital plus centrées sur les affaires internes tels que MadaMasr, Mandara ou encore Wilad el-Balad, pour n'en citer que quelques-unes, peuvent contribuer à renforcer le débat national sur des questions qui auparavant ne bénéficiaient pas d'une attention suffisante. Par ailleurs, en facilitant la récolte d'informations y compris dans des zones jusqu'ici restées en marge de la couverture médiatique, les nouvelles technologies pallient à la centralisation excessive sur le Caire, qui constitue l'un des problèmes fondamentaux de l'information journalistique égyptienne.

\section{Synchronisation et convergence médiatique}

Le système médiatique égyptien, comme d'autres dans la région, est devenu sous bien des aspects un système "hybride » au sein duquel cohabitent les logiques propres aux médias traditionnels et aux nouveaux médias, rendant ainsi la circulation de l'information à la fois plus complexe et plus variée. ${ }^{4}$

4. Andrew Chadwick, 2013, The Hybrid Media System. Politics and Power, Oxford University Press, Oxford. 
La distinction entre anciens et nouveaux médias semble ellemême devenir obsolète, alors que les modalités de production et de distribution de l'information se multiplient.

En Egypte, le phénomène d'hybridation entre les différents médias devient visible dès la moitié des années 2000. Les talk-show télévisés et les journaux se mettent à collecter des informations et des idées sur le web, développant ainsi de véritables "rédactions hybrides $» .^{5}$ Les activistes et les journalistes ayant déjà acquis une certaine visibilité sur le web sont invités à participer aux émissions de télévision ou à écrire directement pour les journaux, faisant ainsi le pont entre les différentes professions. Le quotidien el-Masry el-Youm met sur pied en 2012 en collaboration avec la start-up Meedan une plateforme en ligne de liveblogging. Hisham Kassem, I'un des fondateurs de el-Masry elYoum, travaille depuis des années sur al-Jumhuria al-Jadida, un nouveau journal doté de la première " rédaction convergente » en Egypte. ${ }^{6}$ Le cas Bassem Youssef, dont le programme satyrique a d'abord été diffusé sur Youtube avant d'être reproduit et distribué par $O n T v$, puis par $C B C$, constitue un autre exemple significatif.

Les révoltes de 2011 renforcent l'intégration entre les différentes plateformes. La convergence ne concerne plus seulement les télévisions, les journaux et internet, mais s'étend à d'autres médias. Le collectif Mosireen créé en février 2011 s'occupe non seulement de récolter les vidéos digitales sur la révolution mais il les projette également à Tahrir, inaugurant ainsi une nouvelle forme de cinéma public. Dans cette lignée, la campagne al-'Askar Kazibun (les militaires mentent) organise des projections dans différents quartiers du Caire pour apporter un contrepoint aux récits officiels de l'armée sur les affrontements de la rue Mohamed Mahmoud en novembre 2011.

Enfin, un art de rue tel que les graffitis bénéficie d'une re-médiatisation sur d'autres plateformes. Huguet et Carle, dans un article de ce numéro, qualifient les modalités de production et de circulation des graffitis de la rue Mohamed Mahmoud de «palimpseste ». Les graffitis passent par un processus de production continuel et se trouvent perpétuellement effacés, reproduits et modifiés. Ce phénomène est amplifié et complété par la circulation des images des peintures murales sur les social network, introduisant ainsi un second niveau de diffusion et de re-négociation du

5. Naomi Sakr, "Social Media, Television Talk Shows, and Politica Change in Egypt", Television and New Media, 14:322, 2012.

6. AbdelRahman Mansour et Linda Herrera, 2012, "The Future of Media in Egypt: an Interview with Hisham Kassem", Jadaliyya : http://www.jadaliyya. com/pages/index/7125/the-future-of-media-in-egypt_an-interview-with-his 
signifiant des graffitis, qui se trouvent re-proposés dans des contextes différents de ceux propres au lieu physique où ils ont été réalisés. L'interaction autour de ces images à travers des commentaires, des statuts Facebook et des re-tweet des images rappelle ce que Lawrence Lessig appelle la " culture remix », à savoir une nouvelle modalité d'approche des contenus culturels qu'introduisent les nouvelles technologies digitales. ${ }^{7} \mathrm{C}^{\prime}$ est cette interaction entre, d'un côté, des formes d'expression antique, les peintures murales, et d'autre part les social media, qui selon Carle et Huguet décuple l'efficacité du recours aux graffitis dans le cadre d'une « nouvelle culture contestataire».

Cet exemple, comme d'autres, témoigne des conséquences profondes de la convergence médiatique sur la sphère publique et sur ses rapports avec le champ du politique.

Comme l'affirme Chadwick:

Dans un système médiatique hybride, le pouvoir est exercé par ceux qui parviennent à créer, filtrer ou canaliser le flux d'information de façon à servir leur objectif tout en étant capable de modifier, déclencher ou paralyser l'action d'autres agents, à travers un éventail d'outils médiatiques nouveaux et traditionnels. $^{8}$

Les événements récents en Egypte ont souvent apporté la preuve que les dynamiques "hybrides » propres aux médias introduisent de nouvelles possibilités, à la fois de défier et de s'approprier le pouvoir politique.

Dans certains cas, les nouveaux médias et les médias traditionnels peuvent converger dans une «synchronisation » qui impose des récits à fort impact sur la scène politique. Dans les mobilisations de 2011, les récits révolutionnaires se sont montrés capables d'exploiter de façon efficace tous les types de médias, convergence qui a constitué la clé de formation d'un consensus transversal contre Hosni Moubarak. Toutefois, comme le remarquent Alexander et Aouragh, ${ }^{9}$ la synchronisation entre des typologies de médias différentes reste dépendante des conditions et du contexte politique. Suite à la chute de Moubarak, les médias traditionnels ont généralement ré-adopté une attitude en faveur des autorités au pouvoir, tandis que les mouvements révolutionnaires,

7. Lawrence Lessig, 2008, Remix. Making Art and Commerce Thrive in the Hybrid Economy, Penguin Books, New York.

8. Andrew Chadwick, 2013, The Hybrid Media System. Politics and Power. Oxford University Press, Oxford, p. 207.

9. Anne Alexander et Miriyan Aouragh, 2014, "Egyptıs Unfinished Revolution: the Role of the Media Revisited", International Journal of Communication, 8, p. 890-915. 
renvoyés vers la marge, ont recommencé à exploiter le web 2.0 ou bien les graffitis et le cinéma de rue pour démentir les récits produits par les autorités.

Le 30 juin et le coup d'état qui lui a succédé ont vu s'opérer une autre synchronisation entre différents médias, cette fois en faveur d'un mouvement contre-révolutionnaire soutenu par l'armée et par d'autres composantes de l'ancien régime.

Cette dernière expérience en particulier montre que la convergence et la synchronisation entre différents médias peut donner lieu à de nouvelles méthodes de construction de récits anti-hégémoniques, mais aussi à de nouvelles modalités d'exercice du pouvoir.

\section{La dégénérescence de la sphère publique après la révolution de janvier}

Dans la période qui succède immédiatement à la chute de Moubarak, le système médiatique égyptien semble prêt à vivre une nouvelle phase de son histoire. De nouveaux journaux et chaînes de télévisions font leur apparition, internet se trouve de plus en plus utilisé comme source $d^{\prime}$ information et surtout, l'atmosphère politique rend plus difficile le contrôle sur l'information. La sphère publique n'a jamais été aussi riche et plurielle, et elle semble capable de constituer une garantie à la transition révolutionnaire amorcée par les mobilisations de 2011.

Quelques années plus tard, le scénario semble cependant bien différent. Tous les médias islamistes sont aujourd'hui contraints de fermer. Les journalistes d'al-Jazeera sont arrêtés, puis condamnés en premier jugement à sept à dix années de prison. Les journaux et télévisions privés acceptent de fait de s'aligner sur les positions des autorités au pouvoir, tandis que les voix plus critiques sont éloignées ou contraintes au silence. La censure et l'autocensure reviennent et sont, selon les dires de nombreux journalistes, plus fortes qu'avant la révolution de janvier. A la fin du mois d'octobre 2014, les éditeurs de certains des médias les plus importants publient une déclaration de soutien au gouvernement et s'engagent à ne plus diffuser de nouvelles qui puissent s'avérer délétères dans le cadre de la lutte contre le " terrorisme ». Bien que certaines formes de résistance existent encore sur internet et ailleurs, ${ }^{10}$ le paysage médiatique rappelle plus l'époque de Nasser que celle des dernières années du régime de Moubarak.

10. Par exemple, l'appel lancé sur internet par Khaled el-Balshi dans lequel il critique la position des éditeurs vis-à-vis du gouvernement. L'appel a été signé par des centaines de journalistes. 
Comment, se demande Kai Hafez dans l'article publié dans cette revue, sommes-nous passés en l'espace de quelques mois de la vitalité de la sphère publique en 2011 et 2012, à une situation de contrôle politique aussi radical sur l'information?

Il semble évident que les événements politiques successifs au 30 juin et la forte concentration du pouvoir politique entre les mains des militaires et du régime constituent un facteur important d'explication. Comme le rappelle Gadi Wasfeld, la scène politique en général tend à dominer la scène médiatique. Quand les autorités au pouvoir ne sont ni divisées, ni indécises, la capacité des médias à offrir un contrepoint est réduite, que ce soit en Egypte ou dans les démocraties libérales occidentales. ${ }^{11}$

Toutefois, Kai Hafez rappelle que le système médiatique égyptien contenait déjà en soi certaines limitations endémiques qui ont contribué à sa chute. Dans la lignée des modèles de journalisme proposés par Hallin et Mancini, I'auteur analyse ces limites à travers quatre composantes fondamentales: la relation entre l'Etat et les médias, le degré de parallélisme politique, le développement du professionnalisme journalistique et le marché journalistique. ${ }^{12}$

Revoir brièvement ces points nous permettra de mettre en lumière quelques-uns des éléments fondamentaux du paysage médiatique égyptien en général et de son rapport avec le champ politique.

L'Egypte est encore dominée par les médias d'Etat, lesquels n'ont jamais été réformés de façon à pouvoir offrir un véritable service public comme cela a été fait en Europe. Bien que le sujet fasse depuis longtemps I'objet d'un débat entre les journalistes, ${ }^{13}$ ces derniers ne disposent toujours pas à ce jour d'un plan de réforme possible du secteur public des médias. La constitution de Morsi en 2012 ne s'est pas occupée de résoudre certains des problèmes structurels du secteur, et a de fait encouragé la stagnation de l'organisation du paysage médiatique. La nouvelle constitution en 2014 contient des références explicites à la

11. Gadi Wolfsfeld, 1997, Media and Political Conflict. News from the Middle East, Cambridge University Press, Cambridge.

12. Daniel Hallin et Paolo Mancini, 2004, Comparing Media Systems: Three Models of Media and Politics, Cambridge University Press, Cambridge. 13. Voir à ce propos le chapitre "Stimuli for a Public Service Ethos" in Naomi Sakr, 2013, Transformations in Egyptian Journalism, I.B. Tauris, Londres. 
liberté d'expression ${ }^{14}$, et, du moins sur le papier, fait état d'un progrès par rapport à la constitution précédente. La création d'un Conseil National des Médias en constitue l'un des aspects les plus intéressants: élu par le Parlement, ce dernier devrait effectuer une supervision du système médiatique, tout en élaborant un code d'éthique à destination des journalistes. Toutefois, ces potentielles améliorations se trouvent empêchées de fait par l'ambiguïté qui caractérise la Constitution dans son ensemble. La liste des cas permettant l'inculpation des journalistes devant un tribunal militaire, par exemple, semble avoir été conçue pour permettre d'envisager toutes les situations possibles. ${ }^{15}$

Le second élément est le "parallélisme politique » des médias égyptiens, c'est-à-dire la tendance à produire une information partisane qui épouse des positions politiques spécifiques au détriment de principes journalistiques tels que l'objectivité. Les médias égyptiens présentent ainsi une forme de pluralisme au niveau externe, mais pas au niveau interne: dans leur ensemble, ils représentent des opinions différentes, mais il est rare que ces divergences se trouvent représentées au sein $d^{\prime} u n e$ même télévision ou d'un même journal. Ce phénomène a encouragé une polarisation de la société civile et une radicalisation des positions, comme le montre l'affrontement entre les partisans et les opposants des Frères Musulmans. Le problème, affirme Hafez, est que dans les jeunes démocraties, qui sont caractérisées par une instabilité plus grande, le parallélisme politique peut constituer un obstacle à la formation du consensus nécessaire à l'élaboration d'institutions communes.

Le troisième élément concerne la relation entre l'économie et les médias. En Egypte, la concentration du capital qui caractérise la société dans son ensemble se reflète également sur la répartition des parts dans les industries de la communication. Les médias privés sont tous entre les mains d'un nombre réduit d'hommes d'affaires proches du régime, et la difficulté de générer des profits grâce à la publicité où à la vente d'exemplaires papier rend plus difficile encore l'émergence d'éditeurs "purs », c'est-à-dire n'ayant pas d'intérêts spécifiques dans d'autres secteurs économiques. L'expérience de Ibrahim Eissa, co-fondateur de la chaîne de télévision al-Tahrir, est exemplaire de ce point de vue. Le journaliste décide en octobre 2011 de vendre ses parts de la chaîne, fondée quelques mois auparavant, à deux hommes d'affaires. Peu de

14. L'article 71 déclare $q u^{\prime}$ « il est interdit de censurer, confisquer, suspendre ou fermer des journaux et des médias égyptiens, de quelque manière que ce soit. Exceptionnellement, une censure limitée pourra être appliquée en temps de guerre ou de mobilisation générale » : http://www.constitutionnet.org/files/final_constitution_-idea-_english-2_dec_2013-signed.pdf

15. Voir sur ce point Basil El-Dabh, 2014, "Egyptss Media Crackdown and the New Constitution", Timep : http://timep.org/analysis/media-crackdown 
temps après, ces mêmes parts passent aux mains de Souleiman Amer, un homme d'affaire proche de Hosni Moubarak. Le cas de Hisham Kassem illustre également la difficulté qu'il y a à mettre en place un média indépendant: ce dernier tente en vain depuis des années de convaincre des entrepreneurs privés d'investir dans son journal, alGumhuriya al-Jadida. L'une des raisons principale de son échec est que Kassem impose comme condition fondamentale qu'aucun des actionnaires ne dépasse le seuil de $10 \%$ des parts de la société, afin d'éviter toute ingérence dans la politique éditoriale du journal.

Enfin, le dernier élément concerne la culture professionnelle des journalistes. L'absence de syndicats de presse indépendants, et l'incapacité des syndicats existants à protéger les journalistes des pouvoirs publics ont empêché, y compris après la révolution, le développement d'un professionnalisme indépendant et basé sur des règles éthiques bien définies.

En dernière analyse, le modèle journalistique égyptien peut se comparer aux modèles méditerranéens décrits par Hallin et Mancini, se révélant particulièrement inapproprié dans un contexte non démocratique et instable comme celui de l'Egypte contemporaine.

Si l'article de Kai Hafez met en lumière les défauts endémiques du système médiatique égyptien, les contributions de Bachir Ben Aziz et Marianna Ghiglia s'arrêtent sur le rôle des médias privés comme source de renouvellement de la production journalistique, dans la mesure où ces derniers repoussent les frontières du dicible et offrent de nouvelles perspectives de mise en récit de la réalité.

Le premier article apporte en effet un éclairage sur l'introduction par les médias privés de nouvelles modalités d'organisation du travail journalistique et de production de l'information. L'espace occupé par les médias privés est forcément différent de celui dont bénéficient les organes publics: non seulement ils disposent d'un accès réduit aux sources politiques et aux événements officiels, mais ils sont contraints de travailler avec une génération de journalistes plus jeunes et, pour constituer une véritable concurrence, d'identifier des thèmes et des problématiques auxquelles les médias publics accordent une attention moindre.

Ces éléments, qui sont étroitement liés au travail journalistique en soi plus qu'à un choix de politique éditoriale, ont favorisé les médias privés comme outil privilégié pour la production de nouvelles représentations de la réalité, y compris des mouvements de protestation. Ainsi, ce sont des modalités différentes d'organisation du travail journalistique qui ont permis d'ébaucher les « itinéraires d'action collective » qui ont inspiré les protestations ouvrières en Egypte dans la seconde moitié des années 2000. 
L'article de Marianna Ghiglia examine par ailleurs l'expérience du quotidien al-Badil: Né en 2007, "ni journal partisan, ni journal privé ", al-Badil est un cas presque unique dans I'histoire des journaux égyptiens. Contrairement aux autres médias privés, il s'agit d'un journal conçu dès sa création comme un acteur politique, voué à exercer un militantisme d'opposition qui ne réussit pas à s'exprimer à travers une organisation de parti.

\section{Repenser le rôle des nouvelles technologies}

Le rôle des nouvelles technologies dans ce qu'on appelle le «Printemps Arabe » a été l'un des sujets les plus soumis à controverse ces dernières années, aussi bien dans les médias que dans les cercles universitaires. Jusqu'à ce jour, comme l'écrit Yves Gonzalez-Quijano dans son article, le débat sur les effets des nouvelles technologies sur les processus socio-politiques dans la région offre peu de certitudes, en partie à cause de l'opposition entre cyber-optimistes et cyberpessimistes qui continue de le caractériser.

L'article de Gonzalez-Quijano passe en revue les principaux éléments de la discussion autour de ce qu'on appelle la "Révolution Facebook», essayant d'établir un bilan du rôle des nouveaux médias dans le cadre des révoltes de 2011 et au cours des années qui ont suivi. En parallèle, il invite à examiner les effets des nouvelles technologies sur le long terme, au lieu de s'arrêter, comme cela a été presque toujours le cas aujourd'hui, sur le rapport entre le web 2.0 et les mobilisations de rue.

Pour analyser ces processus, il est donc nécessaire de commencer par se débarrasser d'un déterminisme technologique qui a montré qu'il portait peu de fruits.

Comme le décrit Hanan Badr dans sa contribution à cette revue, les effets des social network dépendent de facteurs structurels et culturels liés au contexte observé. La culture politique en Egypte se trouve relayée dans les modalités d'usage des social network, faisant ainsi obstacle à la possibilité de les utiliser comme instruments révolutionnaires. Une barrière supplémentaire est constituée par les facteurs structurels, des architectures virtuelles utilisées aux institutions gouvernementales qui font usage d'internet de manière de plus en plus efficace. En ce sens, pour reprendre l'expression de Dutton, internet ne constituerait pas un nouveau médium du "quatrième pouvoir ", mais pourrait plutôt s'assimiler à un " cinquième pouvoir »: un espace de relations contesté par des acteurs et des pouvoirs différents, et dont les effets restent à déterminer. ${ }^{16}$

16. William H. Dutton, 2009, "The Fifth Estate Emrging through the Network of Networks", Prometheus: Critical Studies in Innovation, vol. 27, nº 1, pp. 15. 
Une « dévaluation » des dynamiques au sein des réseaux du cyberactivisme pourrait donc fournir le point de départ pour approcher ces thèmes de manière plus efficace. Dans ce but, l'intégration des théories des nouveaux médias à des approches ethnographiques, sociologiques et anthropologiques comme le font certains des articles contenus dans ce numéro pourra constituer un antidote afin, comme l'affirme Myriam Aouragh, "de s'éloigner des conjectures sur l'universalité des expériences digitales $»{ }^{17}$

Comme l'écrit dans son article Enrico de Angelis, nous savons aujourd'hui que les réseaux de l'activisme ne sont pas intrinsèquement démocratiques, mais que des formes de pouvoir et d'inégalités émergent au sein des espaces en ligne, de la même manière que ce qui se passe dans les espaces hors ligne; que les contenus qui circulent sur le web peuvent facilement se trouver falsifiés dans le champ politique; que les network font preuve d'une certaine fragilité quand il s'agit de se confronter, comme on l'a vu pour les compétitions électorales en Egypte et en Tunisie, à des organisations plus structurées. Et surtout, il faut reconnaître que la société tend à transformer internet, plus rapidement que le contraire.

Pourtant, tout en prenant acte des limites de l'activisme en ligne, il est impossible d'ignorer les nombreux cas où ces derniers ont généré des expériences politiques et sociales innovantes avec un impact aboutissant à des changements concrets. Le forum tunisien SfaxOnline en fournit un bon exemple, tel qu'il est décrit dans l'article de Carboni, Crisponi et Sistu qui clôt ce numéro de Egypte/Monde Arabe.

L'expérience de SfaxOnline met en lumière des modalités d'utilisation d'internet différentes de celles qui se basent sur les réseaux sociaux digitaux, lesquels ont fait l'objet de la majorité des analyses effectuées ces dernières années. Cette expérience témoigne de l'utilisation du web comme instrument d'agrégation et de confrontation collective, dans le cadre d'une communauté locale. Il ne s'agit plus ici de réseaux de liens faibles, qui sont ceux que les social network tendent à créer à travers une interaction en majorité virtuelle: le web émerge au contraire comme une technologie à même de générer de nouvelles pratiques de cohabitation et de confrontation culturelle, au sein d'une communauté dont les frontières sont clairement définies. D'une part, les activités de modération du site peuvent rappeler celles utilisées au sein d'un groupe comme "Nous sommes tous Khaled Said », et comme là, elles se traduisent par l'implication en politique d'individus qui auparavant

17. Miriyam Aouragh, 2012, "Social Media, Mediation and the Arab Revolutions », TripleC, 10 (2), 518-536. 
en étaient exclues. D'autre part, il s'agit d'une plateforme basée sur les liens forts d'une communauté qui existe déjà, mais qui est privée d'autres espaces de confrontation publique. On peut l'interpréter comme un retour vers le passé et vers une époque antérieure aux social network. Et pourtant, repenser les modalités d'utilisation des nouveaux médias peut aussi revenir à cela: écarter ou réinventer des formes d'activités qui se sont révélées non fonctionnelles sur le long terme. 